\title{
ON THE KERNEL OF A TOPOLOGICAL SEMIGROUP WITH CUT POINTS
}

\author{
YATARŌ MATSUSHIMA
}

W. M. Faucett [1] recently studied the structure of the kernel of a compact connected mob which has a point that cuts the kernel. L. W. Anderson [2] has characterized the cut point of a connected topological lattice. The main purpose of this paper is to find a lattice theoretic characterization of the kernel by means of cut points in the topological semigroups derived from topological lattices. Using the concept of $B$-covers [3], we shall define a suitable multiplication in topological lattices to illustrate the structure of the kernel by lattice diagrams. The fact that a special case of Faucett's Theorem (Theorem 2) can be obtained from Anderson's result (Lemma 5) is important.

1. Preliminaries. We recall that a topological lattice is a Hausdorff space, $L$, together with a pair of continuous functions $\wedge: L \times L$ $\rightarrow L$ and $\vee: L \times L \rightarrow L$ which satisfy the usual conditions stipulated for a lattice. If $X$ is a topological space and $p \in X$, we say that $p$ is a cut point of $X$ if $X \backslash p$ is not connected, i.e., if $X \backslash p=U \cup V$ such that $U \neq \square \neq V$ and $U^{*} \cap V=\square=U \cap V^{*}$, where by $A^{*}$ we mean the closure of $A$.

Hereafter let $S$ be a connected topological lattice which satisfies the modular law. Now we introduce a multiplication in $S$ as follows:

(M) $x y=(a \vee x) \wedge(b \vee y)$ for two fixed elements $a, b$ of $S$.

For any two elements $a, b$ of a lattice $S$ let

$$
B(a, b)=\{x \mid(a \vee x) \wedge(b \vee x)=(a \wedge x) \vee(b \wedge x)=x\} ;
$$

then $B(a, b)$ is called the $B$-cover of $a$ and $b[3]$. We define a mob to be a Hausdorff space together with a continuous associative multiplication. Then $S$ is a mob with respect to $(\mathrm{M})$, for the multiplication is continuous since $S$ is a topological lattice and moreover it is associative by Lemma 1 .

2. The kernel $B(a, b)$ of a mob $S$.

Lemma 1. $x(y z)=(x y) z$ in $S$.

Proof. We have

Received by the editors December 12, 1959. 


$$
\begin{aligned}
x(y z) & =(a \vee x) \wedge(b \vee((a \vee y) \wedge(b \vee z))) \\
& =(a \vee x) \wedge(a \vee b \vee y) \wedge(b \vee z) \\
(x y) z & =(a \vee((a \vee x) \wedge(b \vee y))) \wedge(b \vee z) \\
& =(a \vee x) \wedge(a \vee b \vee y) \wedge(b \vee z) \text { by the modular law. }
\end{aligned}
$$

Lemma 2. If $x \in B(a, b), y \in S$, then (i) $x x=x$, (ii) $x y \in B(a, b)$, $y x \in B(a, b)$.

Proof. (i) follows from the definition of $B(a, b)$.

(ii) $(a \vee x y) \wedge(b \vee x y)$

$$
\begin{aligned}
& =(a \vee((a \vee x) \wedge(b \vee y))) \wedge(b \vee((a \vee x) \wedge(b \vee y))) \\
& =(a \vee x) \wedge(a \vee b \vee y) \wedge(a \vee b \vee x) \wedge(b \vee y) \\
& =(a \vee x) \wedge(b \vee y)=x y \text { by the modular law; } \\
& (a \wedge x y) \vee(b \wedge x y) \\
& =(a \wedge((a \vee x) \wedge(b \vee y))) \vee(b \wedge((a \vee x) \wedge(b \vee y))) \\
& =(a \wedge(b \vee y)) \vee(b \wedge(a \vee x)) \\
& =(a \vee(b \wedge(a \vee x))) \wedge(b \vee y) \\
& =(a \vee b) \wedge(a \vee x) \wedge(b \vee y) \text { by the modular law. }
\end{aligned}
$$

Since $x \leqq a \bigvee b$ for $x \in B(a, b)$ we have $(a \bigvee b) \wedge(a \vee x) \wedge(b \vee y)$ $=(a \bigvee x) \wedge(b \bigvee y)=x y$. Similarly we have $y x \in B(a, b)$.

Lemma 3. Let $p \in B(a, b)$; then $S p$ is a minimal left ideal and $p S$ is a minimal right ideal.

Proor. We shall prove that $(x p)(S p)=x p$ for $x \in S, p \in B(a, b)$. For $y \in S$ we have $(x p)(y p)=(x p y) p=((a \bigvee x) \wedge(a \bigvee b \vee p) \wedge(b \vee y)) p$ $=(a \bigvee x) \wedge(a \bigvee b \bigvee p) \wedge(a \bigvee b \bigvee y) \wedge(b \bigvee p)=(a \bigvee x) \wedge(b \bigvee p)=x p$ since $p \leqq a \vee b$. Similarly we have $(p S)(p x)=p x$.

Lemma 4. If $p \in B(a, b)$, then $B(a, b)=S p S$.

Proof. By Lemma 2 we have $B(a, b) \supset S p S$. If we take $r \in p S \cap S q$ for $q \in B(a, b)$, then we have $q r=q$ by Lemma 3, where $r=p x$ for some $x \in S$. Accordingly we have $B(a, b) \subset S p S$.

As a consequence, we have the following theorem.

THEOREM 1. $B(a, b)$ is the kernel of $a$ mob $S$.

3. The structure of the kernel $B(a, b)$ with cut points.

Lemma 5 (L. W. ANDERson). If $S$ is a connected topological lattice and if $p \in S$ then $p$ is a cut point of $S$ if, and only if, $p \neq 0, p \neq I$ and $L=(p \bigvee L) \cup(p \wedge L)$. 
The next theorem is a special case of Faucett's theorem [1, Theorem 1.3].

THEOREM 2. Let $S$ be a compact connected mob derived from a compact connected topological lattice introducing the multiplication (M) into it. If there exists $a$ point $p \in S$ that cuts $B(a, b)$, then we have either

(i) $B(a, b)=\{x \mid a \leqq x \leqq b\}=S p$, that is, $B(a, b)$ is a minimal left ideal, and every element of $B(a, b)$ is left zero for $S$; or

(ii) $B(a, b)=\{x \mid b \leqq x \leqq a\}=p S$, that is, $B(a, b)$ is a minimal right ideal, and every element of $B(a, b)$ is right zero for $S$.

Proof. Since $p$ cuts $B(a, b)$, we have $B(a, b)=A \cup B$, where $A=\{x \mid x \leqq p\}$ and $B=\{x \mid x \geqq p\}$, by Lemma 5 .

Now suppose that $a, b \leqq p$; then for any element $x \in B$ such that $x>p$, we have $(a \wedge x) \bigvee(b \wedge x)=a \bigvee b \leqq p<x$, that is, $x$ does not belong to $B(a, b)$, a contradiction. Similarly the case where $a, b \geqq p$ does not occur. Thus we have either $a \leqq p \leqq b$ or $b \leqq p \leqq a$. In the first case, any element $x$ such that either $x<a$ or $b<x$ does not belong to $B(a, b)$. Now we shall prove that $B(a, b)=\{x \mid a \leqq x \leqq b\}=S p$. Let $p \in B(a, b)$, $x \in S$; then $x p=(a \bigvee x) \wedge(b \bigvee p)=(a \bigvee x) \wedge b=a \vee(b \wedge x)$ by the modular law. Then we have $a \leqq x p \leqq b$, hence $\operatorname{Sp} \subset B(a, b)$.

Conversely, if we take $k \in B(a, b)$, then $k p=(a \bigvee k) \wedge(b \vee p)$ $=k \wedge b=k$ since $a \leqq k, p \leqq b$. It follows that $B(a, b) \subset S p$. Accordingly we have $S p=B(a, b)$, and hence $B(a, b)$ is a minimal left ideal by Lemma 3.

Now let $x \in S, k \in B(a, b)$; then $k x=(a \bigvee k) \wedge(b \bigvee x)=k \wedge(b \bigvee x)=k$ since $k \leqq b$, that is, every element of $B(a, b)$ is a left zero for $S$. This completes the proof of (i). Similarly we can prove (ii).

4. The case where no point cuts the kernel $B(a, b)$ for $S$. Throughout this section we shall assume that there is no point that cuts the kernel $B(a, b)$ of the mob $S$ derived from a topological lattice.

We can easily find that (i) if $a \leqq b$, then $B(a, b)$ is a minimal left ideal for $S$, (ii) if $b \leqq a$, then $B(a, b)$ is a minimal right ideal for $S$, (iii) if $a, b$ are noncomparable, then $B(a, b)$ has the same structure as that in Lemma 4.

Let us define a two-sided ideal $T$ of a mob $S$ to be a prime ideal provided that whenever $S \backslash T$ is non-null then $S \backslash T$ is a submob. A submob in a mob $S$ is a nonvoid set $T$ contained in $S$ such that $T T \subset T$. Now we shall find a necessary and sufficient condition for a two-sided ideal $C$ containing $B(a, b)$ to be a prime ideal in the case where $S \backslash z$ $=C \cup D, C \neq \square \neq D$ and $C^{*} \cap D=\square=C \cap D^{*}$. In this case we do not assume that $S$ is connected. 
Lemma 6. Let $S \backslash B(a, b) \ni z$; then $z z \in B(a, b)$ if, and only if, $z \leqq a \bigvee b$.

Proof. By the modular law, we have $(a \bigvee z z) \wedge(b \bigvee z z)=z z,(a \wedge z z)$ $\vee(b \wedge z z)=(a \bigvee z) \wedge(b \bigvee z) \wedge(a \bigvee b)$. If $z \leqq a \bigvee b$, then we have $z z$ $\in B(a, b)$. Conversely if $z z \in B(a, b)$, then $z z=(a \bigvee b) \wedge(a \bigvee z) \wedge(b \vee z)$ $=(a \bigvee b) \wedge z z$, and hence $z \leqq(a \bigvee z) \wedge(b \bigvee z)=z z \leqq a \bigvee b$. Hence we have $z \leqq a \bigvee b$.

THEOREM 3. Let $S$ be a mob with respect to multiplication (M), and let $z$ be an element of $S$ such that $S \backslash z=C \cup D, C \neq \square \neq D, C^{*} \cap D=\square$ $=C \cap D^{*}$ and $C$ is a two-sided ideal containing $B(a, b)$; then $C$ is a prime ideal if, and only if $z>a \bigvee b$.

Proof. By Lemma 5, we have either (i) $y<z<x$ or (ii) $y>z>x$ for all $x \in C, y \in D$. If $z>a \bigvee b$, let $S \backslash C=\{z, D\}=T \ni y_{1}, y_{2}$; then $y_{1}, y_{2}$ $\geqq z>a \bigvee b$, and hence $y_{1} y_{2}=\left(a \vee y_{1}\right) \wedge\left(b \bigvee y_{2}\right)=y_{1} \wedge y_{2} \geqq z>a \vee b$, that is, $y_{1} y_{2} \in T$. Then $C$ is a prime ideal. If $z \ngtr a \bigvee b$, then $z \leqq a \bigvee b$ by Lemma 5. It follows that $z z \in B(a, b) \subset C$ by Lemma 6 , and then $C$ is not a prime ideal. This completes the proof.

\section{REFERENCES}

1. W. M. Faucett, Topological semigroups and continua with cut points, Proc. Amer. Math. Soc. vol. 6 (1955) pp. 748-756.

2. Lee W. Anderson, On the distributivity and simple connectivity of plane topological lattices, Trans. Amer. Math. Soc. vol. 91 (1959) pp. 102-112.

3. Y. Matsushima, On the B-covers in lattices, Proc. Japan Acad. vol. 32 (1956) pp. 549-553.

Gunma University, MaEbashi, Japan 